\title{
Applications of a high-voltage SMPS in water disinfection
}

\author{
P.T.Johnstone and P.S.Bodger
}

\begin{abstract}
Microorganisms suspended in water can be destroyed by the application of short duration high-magnitude electric fields. A $50 \mathrm{~Hz}$ high-voltage water treatment device has been previously presented. The application of a switch-mode power supply (SMPS) to provide the high voltage required by this device is examined. The SMPS has a number of significant advantages over a $50 \mathrm{~Hz}$ supply. It is able to reduce the operating energy requirements by over $90 \%$. It is also smaller, more lightweight and less expensive to manufacture. The effectiveness of the SMPS in reducing numbers of bacteria is tested. The SMPS is not as effective as the $50 \mathrm{~Hz}$ supply; however, this may be compensated by using multiple electrodes in series.
\end{abstract}

\section{Introduction}

The destruction of pathogenic or harmful microorganisms in water is important to provide supplies that are potable. The provision of potable water supplies will lessen the risks of disease caused by contaminated water.

Microorganisms can be destroyed by rupturing their membranes with an electric field. The membrane acts as a dielectric, which charges up on application of an external field. Disruption of the membrane occurs when the electric field strength reaches some critical value. This idea is currently being applied to the disinfection of various types of liquid foods, including milk and fruit juice [1-6]. This application is termed 'pulsed electric field treatment' (PEF). PEF treatment involves charging storage capacitors to a high voltage and subsequently discharging them into the liquid load, using a high-voltage switch.

The process of cell inactivation depends upon both applied field strength and exposure time. Increasing either the electric field strength or exposure time increases the amount of inactivation. The most effective voltage waveform would thus be DC. However, DC waveforms pose electrolysis problems (the electrode surface quickly degrades), present greater risk in terms of operator safety and system insulation, and are less robust to the generation of air bubbles and arcing between electrode surfaces. Higher frequency waveforms reduce most of these problems, but have less effect on the reduction of bacteria.

The authors have previously described a new type of water disinfection device that uses high-magnitude electric fields to destroy the viability of microorganisms suspended in water $[7,8]$. A continuous high-voltage $50 \mathrm{~Hz}$ waveform is applied across a set of parallel electrodes through which the water flows. As the water passes through the electrodes, the high electric field ( $30 \mathrm{kV} / \mathrm{cm}$ RMS) ruptures the mem-

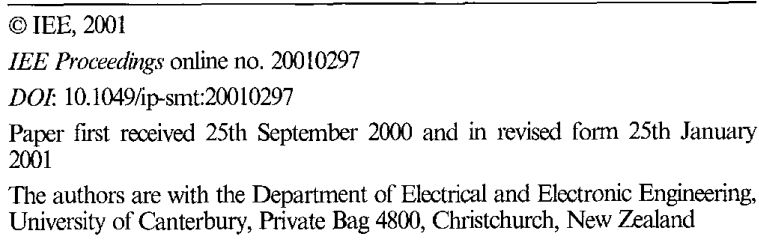

branes of bacteria and other living cells, causing them to become non-viable.

The applied voltage waveform used by these initial highvoltage water treatments was $50 \mathrm{~Hz} \mathrm{AC} .50 \mathrm{~Hz}$ was used because of its ready availability (national power distribution frequency), and because of its ability to reduce electrolysis problems.

The device utilises a mixed bed deionising resin, which removes ions and charged particles from the water. This is necessary in order to increase its resistivity and enable the high electric field to be placed across it. The high resistivity of the deionised water means that the electrical power used by the device is low. The initial disinfection device used only $40-60 \mathrm{~W}$ to disinfect a flow of one litre per minute [7].

Design changes were made to the device to allow for commercialisation, without adversely affecting its disinfection capability [8]. The main changes were to allow for mass construction of the electrodes and air trap by injection moulding. The commercial prototype was tested successfully on common bacteria, Serratia marcescens, and pathogenic protozoa, Giardia intestinalis [8].

The high voltage disinfection device described by the authors uses an exposure time of $17 \mathrm{~ms}$ [8]. The reason for the relatively large exposure time is due to the $50 \mathrm{~Hz}$ sinusoidal waveform. There are regions in any sinusoidal waveform where the voltage is low (either side of a zero crossing). The field strengths in these regions may be below the critical strength $\left(E_{c}\right)$ needed for membrane disruption. Thus, the exposure time must be large enough to ensure all water has been exposed to a high voltage peak.

This paper explores the advantages that can be obtained by using a switch-mode power supply (SMPS) to replace the $50 \mathrm{~Hz}$ high-voltage supply. A high-frequency SMPS may further reduce electrolysis while generating an alternating high-voltage DC (bipolar square wave). Low electric field regions in the waveform are dramatically reduced. The SMPS thus retains some of the advantages of a DC supply, without its disadvantages.

\section{Apparatus}

A high-voltage SMPS was constructed that provides a maximum RMS value of $3 \mathrm{kV}$ chopped $\mathrm{DC}$ at $17 \mathrm{kHz}$ 


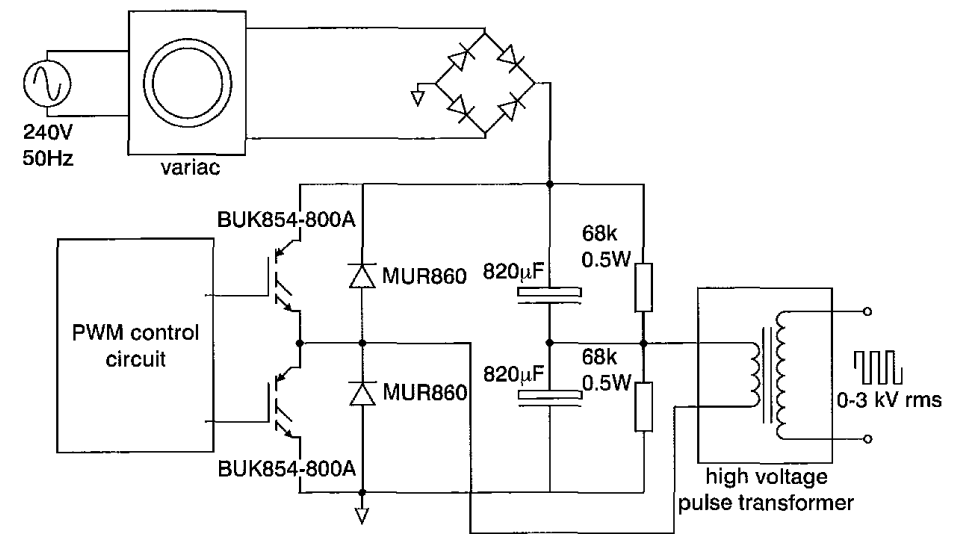

Fig. 1 High-voltage switch-mode power supply

(Fig. 1). A variac was used to provide a single phase $50 \mathrm{~Hz}$ variable input $(0-230 \mathrm{~V})$. The SMPS rectifies the input voltage to provide a $0-320 \mathrm{~V}$ DC bus. Two IGBT switches (BUK854-800A) operating in a half-bridge configuration chop the DC bus. The resulting $17 \mathrm{kHz}$ chopped waveform is fed through a step-up pulse transformer to increase its magnitude by a factor of 9.4 . The $0-3 \mathrm{kV}$ chopped waveform is applied directly to the electrode chamber. The power rating of the SMPS is 400VA.

An electrode chamber was constructed using $5 \mathrm{~mm}$ stainless steel (grade 316T) bar as the electrode material. These bar electrodes were inserted into a machined piece of acetal plastic, with a $5 \mathrm{~mm}$ diameter hole drilled perpendicular to the electrode axis (Fig. 2). The electrode surfaces were polished and the edges slightly burred to reduce irregularities that may cause high electric field stress.
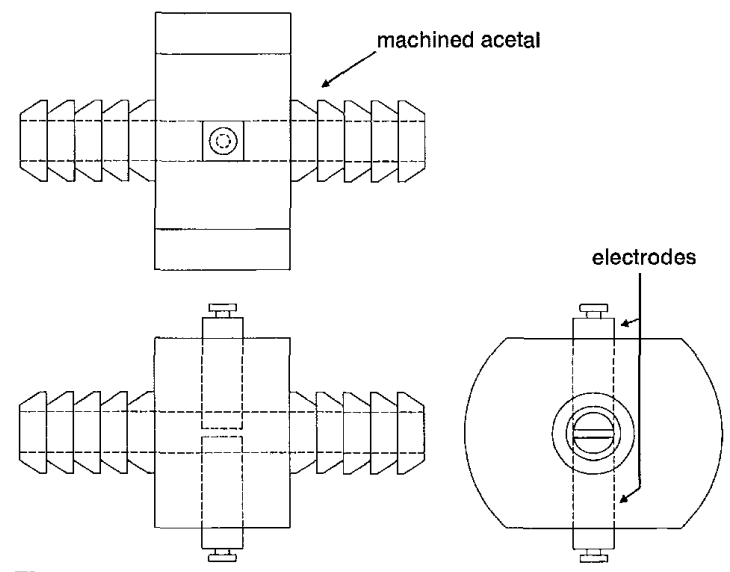

Fig.2 Electrode chamber

Electrodes: $5 \times 5 \mathrm{~mm}$ bar, stainless steel $316 \mathrm{~T}$

The electrodes press-fit into the acetal body. The gap between electrode surfaces is readily adjustable by using metal feeler gauges of various thicknesses. This gap, through which the water passes, determines the electric field strength for a given applied voltage. The gap used throughout these expenments was $0.65 \mathrm{~mm}$.

\section{Microbiology}

Two types of bacteria were used in this experiment. Serratia marcescens was used in the $50 \mathrm{~Hz}$ treatment device because of its small size and its red pigment, which makes it easy to identify $[7,8]$. The organisms were cultured in standard nutrient broth (NB) and incubated at $30^{\circ} \mathrm{C}$.
Escherichia coli was also used in this experiment because of its relevance to water quality. $E$. coli is a member of the faecal coliform family. Coliforms are used in water supplies to indicate a source of faecal contamination and the possible presence of other pathogens. E. coli is a larger bacterium than $S$. marcescens. Thus, it may be more susceptible to the high voltage since the induced transmembrane potential is directly proportional to the radius of the cell. E. coli was also cultured using standard nutrient broth (NB), and incubated at $37^{\circ} \mathrm{C}$.

For both organisms, the inoculated broth was incubated overnight until reaching the early stationary growth phase. The bacteria were harvested by centrifugation and resuspended in sterile deionised water immediately prior to treatment. The starting concentration of bacteria in each experiment was between $1 \times 10^{5}$ and $3 \times 10^{5}$ colony forming units (CFU)/ml.

Bacteria suspensions were added to 1.21 of deionised water and forced through the electrode chamber by a small submersible pump (Fig. 3). An adjustable in-line valve enabled the flow rate to be varied. Treated and non-treated samples were taken immediately at the outlet of the electrode chamber. Each sample was collected with the electric field strength and flow rate adjusted to the correct value. The electric field was disabled before taking a control immediately after each sample.

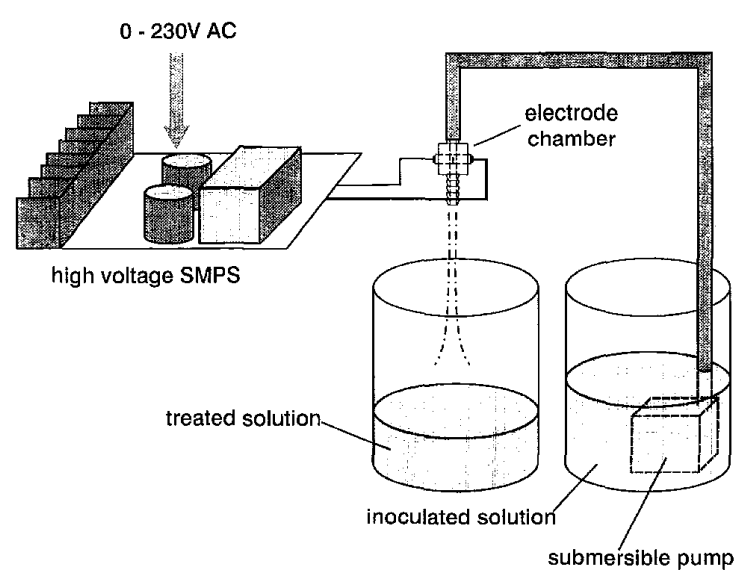

Fig.3 Experimental setup

Viability of the bacteria before and after treatment was assayed by counting colonies on an agar plate. The samples were first serially diluted with sterile deionised water and a known amount spread onto NA agar plates. The plates were incubated for $24 \mathrm{~h}$ at the respective incubation temperature. The number of colony forming units (CFUs) was 
then counted. The dilutions were carried out in such a way as to give between 20 and 300 colonies per plate (except for very low concentration samples that had zero or very few colonies). Duplicate plates were used for each dilution.

\section{Results}

The survival ratio of $S$. marcescens was measured for an electric field strength of $30 \mathrm{kV} / \mathrm{cm}$, and varying exposure time (Fig. 4). As exposure time is increased, the survival ratio decreases. However, the survival ratio decrease is not large despite a relatively large change in exposure time. The shortest exposure time is $0.34 \mathrm{~ms}$, and this gives a survival ratio of 0.05 ( $95 \%$ lysing).

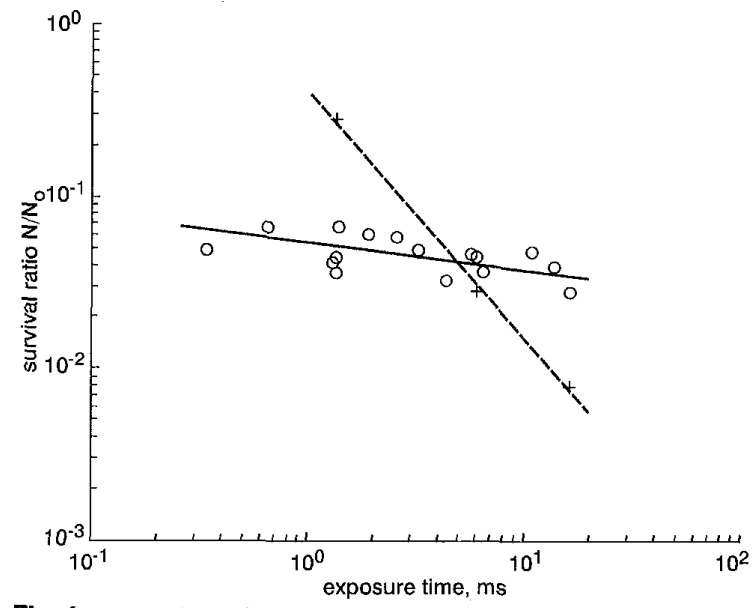

Fig. 4 Survival ratio for S. marcescens at $30 \mathrm{kV} / \mathrm{cm}$ field strength Electrodes: $0.65 \times 5 \times 5 \mathrm{~mm}$, stainless steel

$-50 \mathrm{~Hz}$

Fig. 4 also shows the performance comparison between the SMPS and $50 \mathrm{~Hz}$ [7]. At high exposure times the $50 \mathrm{~Hz}$ waveform has a greater lysing effect. At low exposure times the SMPS waveform is more effective. The low field regions in the $50 \mathrm{~Hz}$ waveform reduce the effectiveness of this waveform at short exposure times.

\subsection{Results from E. coli testing}

SMPS single application experiments were repeated on $E$. coli. Fig. 5 shows the results in comparison with $S$. marcescens. The tests show a similar survival ratio for each type of bacteria.

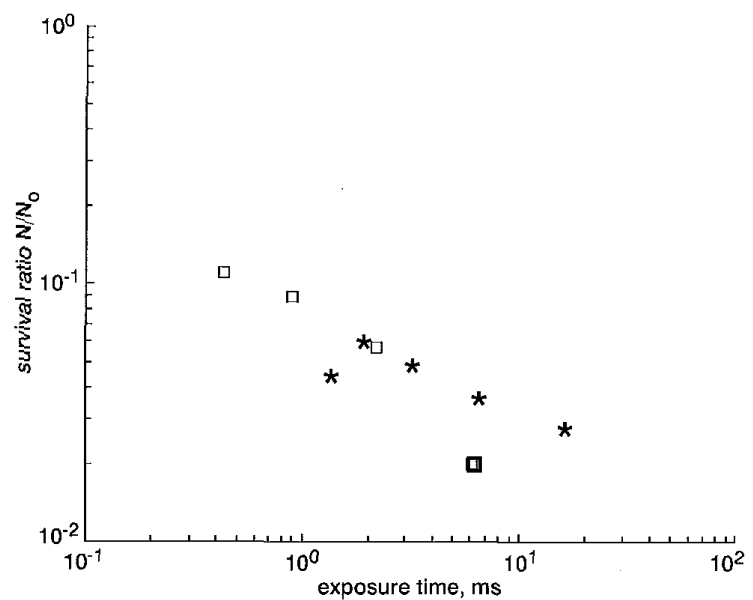

Fig. 5 Survival ratio for $E$, coli and $S$. marcescens at $30 \mathrm{kV} / \mathrm{cm}$ Electrodes: $0.65 \times 5 \times 5 \mathrm{~mm}$, stainless steel $17 \mathrm{kHz}$ SMPS

* S. marcescens

IEE Proc:-Sci. Meas. Technol, Vol. 148, No. 2, March 2001

\subsection{Effect of overshoot}

The water load being used has a high resistance since it is deionised. The water load also has a capacitance, but since the impedance due to this at the operating frequency is much higher than that of the resistance, it has very little effect. With this high-impedance load a large amount of overshoot and ringing occurs in the SMPS waveform (Fig. 6a). The ringing is caused by distributed capacitance and leakage inductance in the high-voltage transformer. The transformer is underdamped. The damping factor of a pulse transformer may be described by eqn. 1 [9].

$$
\delta=\frac{\sqrt{a}}{2}\left(\frac{R_{1}}{Z_{0}}+\frac{Z_{0}}{R_{2}}\right)
$$

where $Z_{0}=\sqrt{ }\left(L_{/} / C\right)$ is the characteristic impedance of the transformer, $R_{1}$ is the total source impedance (primary winding resistance + external source impedance), $R_{2}$ is the sum of the load resistance and the secondary winding resistance referred back to the primary, and $a$ is the attenuation factor, defined as $a=R_{2} /\left(R_{1}+R_{2}\right) . L_{l}$ is the leakage inductance of the transformer and $C$ is the distributed capacitance referred to the primary. A transformer with $\delta$ $<1$ is highly damped, $\delta=1$ is critically damped, and $\delta>1$ is underdamped [9].
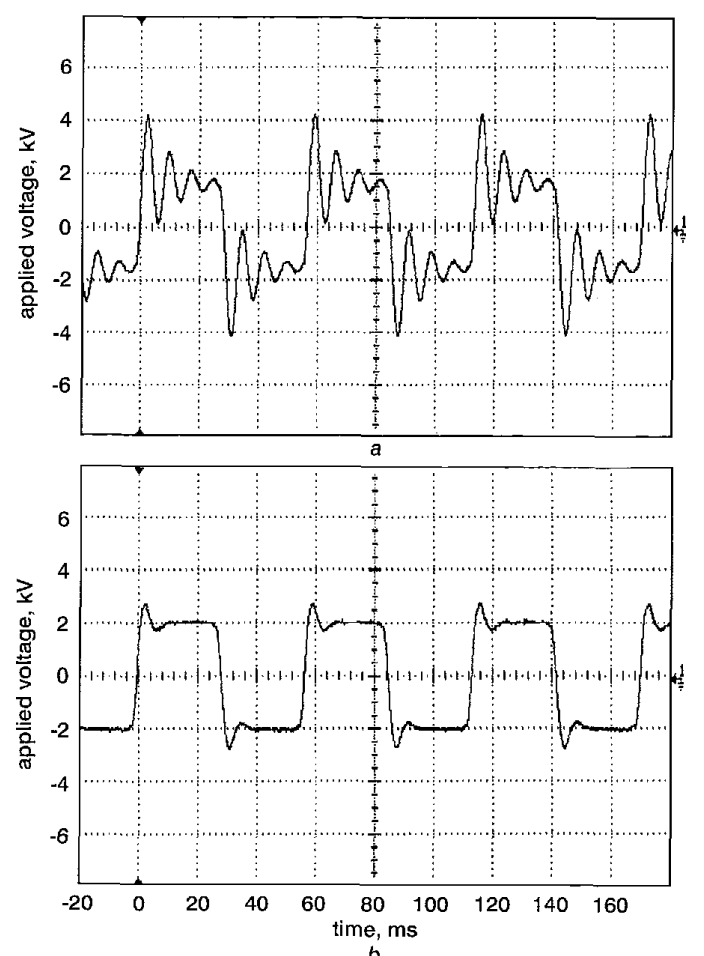

Fig.6 SMPS wiveforms

Underdamped (> 100\% overshoot)

$b$ Damped by $11 \Omega$ source impedance ( $20 \%$ overshoot)

It can be seen from eqn. 1 that overshoot and ringing on the SMPS waveform may be damped by the addition of transformer source resistance. Fig. $6 b$ shows the damped waveform when a resistance of $11 \Omega$ is placed in series with the transformer primary.

To determine if the high-voltage overshoot had any effect on the lysing of bacteria, experiments were performed using both waveforms. Fig. 7 shows the results of this testing on S. marcescens. The waveform with the high overshoot had more effect in lysing bacteria than the one with a low overshoot. The difference, however, is not great. The waveform 
with little overshoot increased the survival ratio by an average of $0.2 \mathrm{log}$. It would seem that the level of damping does not make a significant difference to the survival ratio.

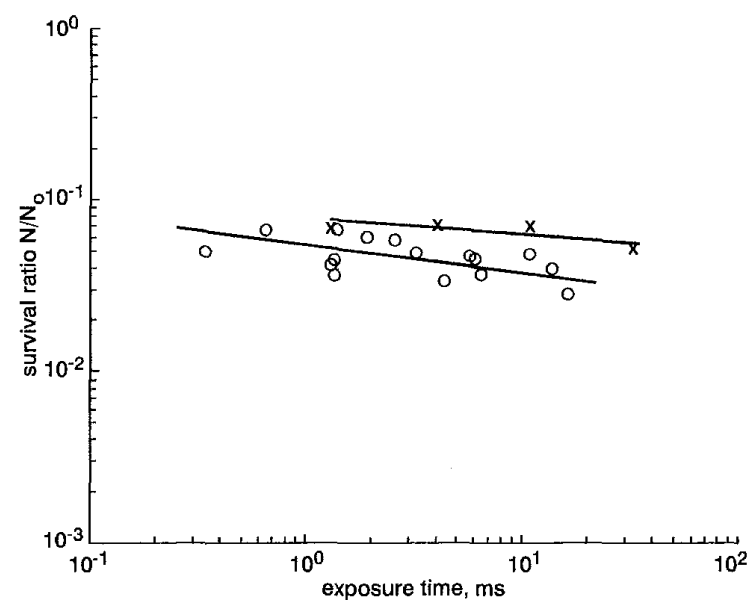

Fig.7 Effect of overshoot on survival ratio of S. marcescens Electrodes: $0.65 \times 5 \times 5 \mathrm{~mm}$, stainless steel $30 \mathrm{kV} / \mathrm{cm}$ RMS $\mathrm{O}>100 \%$ overshoo $\times 20 \%$ overshoot

A disadvantage of the waveform with high overshoot and ringing is the instability of the circuit. The high-voltage overshoots increase the stress on the transformer insulation, and also the electrode stability. That is, the transformer operational lifetime may be reduced because of the insulation stresses, and the electrodes may be more prone to arcing and damage due to the high-voltage stress.

In these experiments a primary series resistance was used to provide the damping. This method is inefficient and dissipates a large amount of power, which increases as the amount of current drawn increases. For this reason, a series resistance is not practical for a working model disinfection unit. A better way of providing damping would be to optimise the transformer leakage inductance and distributed capacitance.

\subsection{Effects of variations in electric field strength}

The electric field strength was varied to ascertain its effect on survival ratio (Fig. 8). It can be seen that as the field strength is increased, the survival ratio decreases. As

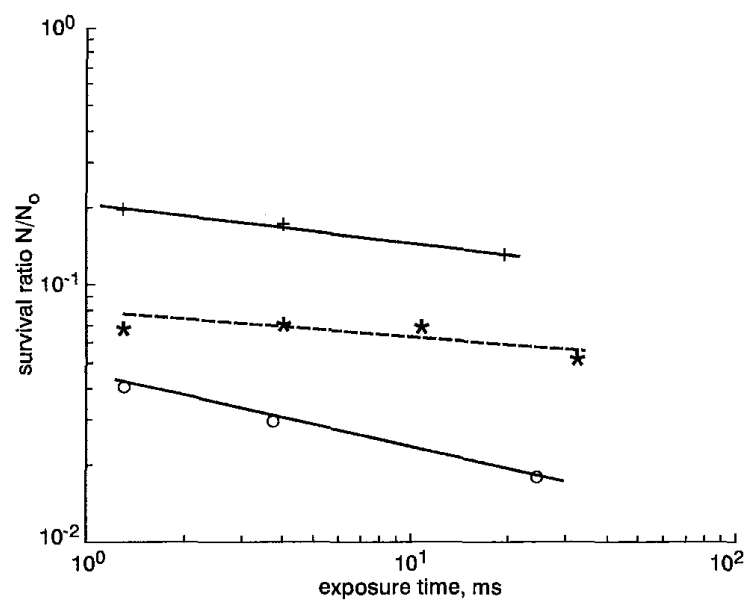

Fig.8 Effect of electric field sirength on survival ratio of $S$. marcescens Electrodes: $0.65 \times 5 \times 5 \mathrm{~mm}$, stainless steel

$17 \mathrm{kHz}$ SMPS, $20 \%$ overshoot

$+20 \mathrm{kV} / \mathrm{cm}$

* $30 \mathrm{kV} / \mathrm{cm}$

$40 \mathrm{kV} / \mathrm{cm}$ expected, higher field strengths are more effective in reducing the numbers of bacteria. For an exposure time of $1.3 \mathrm{~ms}$, the $20 \mathrm{kV} / \mathrm{cm}$ waveform survival ratio was $0.2(80 \%$ lysing), as compared with the $40 \mathrm{kV} / \mathrm{cm}$ survival ratio of 0.04 (96\% lysing).

\subsection{Multiple applications}

The overall efficiency of the treatment device may be increased by operating at a lower exposure time and/or voltage magnitude. The corresponding decrease in lysing rate may be compensated by the addition of a multiple number of electrodes.

Some multiple application tests were undertaken on both E. coli and S. marcescens to prove this idea (Fig. 9). The E. coli results show that successive treatments further reduce the survival ratio. However, there also seems to be a limit to the survival ratio, at which further applications have no added effect. The survival ratio limit for $E$. coli is about 0.01 ( $99 \%$ lysing rate). A similar limit is reached when multiple application tests are run on $S$. marcescens. The reason for the survival ratio limit is unclear, although a similar effect was seen in the initial $50 \mathrm{~Hz}$ treatment results [8].

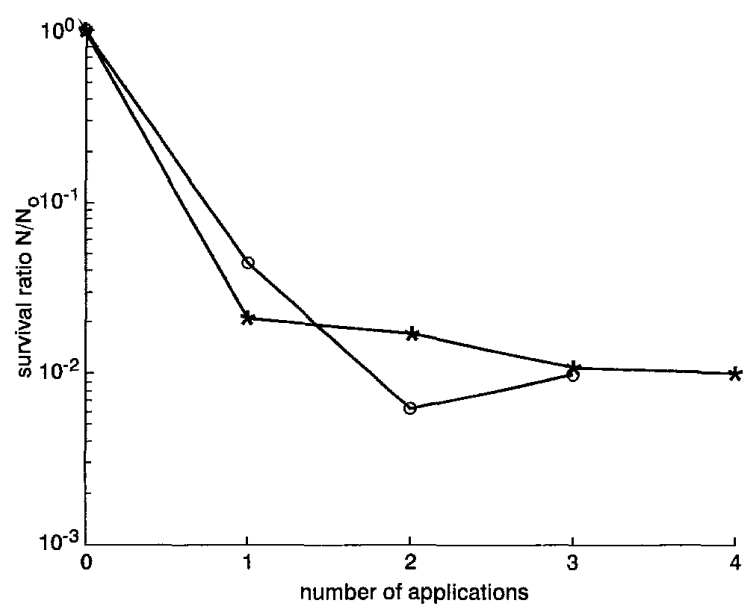

Fig.9 Multiple applications of SMPS treatment at $30 \mathrm{kV} / \mathrm{cm}$ $\begin{array}{ll}-+- & \text { S. marcescens } \\ -\mathrm{O} & \text { E. coli }\end{array}$

\section{Discussion}

Although the SMPS is not as effective in reducing the numbers of bacteria as the previously cited $50 \mathrm{~Hz}$ model, it does have significant advantages. The high-voltage SMPS is much smaller, lightweight and cheaper to manufacture than its $50 \mathrm{~Hz}$ counterpart. The higher frequency used in the SMPS is electrically more stable than $50 \mathrm{~Hz}$ (i.e. the electrodes are much more robust to arcing). The SMPS does not need an air trap before the electrodes, whereas this is critical to the $50 \mathrm{~Hz}$ device. Because the SMPS does not have 'dead' areas of low electric field, the exposure time and thus power may be reduced. Most of the experiments described in this paper used an exposure time of $1.3 \mathrm{~ms}$, whereas the $50 \mathrm{~Hz}$ treatment uses $17 \mathrm{~ms}$. This $92 \%$ reduction in exposure time is equivalent to a $92 \%$ reduction in energy or power used per unit volume of water. The reduction in energy needed to treat water may contribute to lower operating costs, but also may allow water of higher conductivity to be treated.

The lower performance of the SMPS in reducing the numbers of bacteria may be partially compensated by the use of multiple treatments (i.e. two electrodes in series). 
This will bring the performance level closer to the $50 \mathrm{~Hz}$ performance level while still maintaining the lower energy levels and other benefits.

The tests described in this paper that use both the highvoltage SMPS and $50 \mathrm{~Hz}$ technology, are tests of the ability of the electric field to destroy the viability of bacteria. When used as part of a commercial device, however, a chemical deionising resin may be necessary. The deionising resin can itself act as a filter and remove negatively-charged bacteria from the water. For this reason the overall bacteria removal rates of a commercial system may be higher than these tests have shown.

\section{Conclusions}

A high-voltage water treatment device that operates at $50 \mathrm{~Hz} \mathrm{AC}$ has previously been presented. This device uses a $50 \mathrm{~Hz}$ step-up transformer to provide the high voltage required. The large size and weight of this transformer has prompted design of a SMPS to replace it.

A high-voltage SMPS was designed for use in water disinfection. The SMPS has a number of significant advantages over the previous $50 \mathrm{~Hz}$ treatment device. It is able to reduce operating energy requirements by over $90 \%$. It is also smaller, more lightweight and less expensive to manufacture.

The high-voltage SMPS has been successfully tested on the bacteria $S$. marcescens and $E$. coli. The SMPS does not reduce the survival ratio of bacteria as much as the $50 \mathrm{~Hz}$ treatment, but the exposure time and associated energy is significantly reduced. Using a number of SMPS treatment devices in series may further reduce the survival ratio.

\section{References}

I MIZUNO, A., INOUE, T., YAMAGUCHI, S., SAKAMOTO, K., SAEKI, T., MATSUMOTO, $Y$. and MINAMIYAMA, $K$.: 'Inactivation of viruses using pulsed high electric field'. Conf. Record of IEEE IAS Annual Meeting, 1990, Vol. 1, pp. 713-719

2 JAYARAM, S., CASTLE, G.S.P., and MARGARITIS, A.: 'Effects of high electric field pulses on Lactobacillus brevis at elevated temperatures'. Conf. Record of IEEE IAS Annual Meeting, 1991, pp. 674 681

3 OIN, B.L., BARBOSA-CANOVAS, G.V. SWANSON, B.U, PEDROW, P.D., and OLSEN, R.G.: 'A continuous treatment system for inactivating microorganisms with pulsed electric fields' Conf. for inactivating microorganisms with pulsed electric fields' Conf.
Record of IEEE IAS Annual Meeting, 1995, Vol. 2, pp. 1345-1352

4 MAZUREK, B., LUBICKI, P., and STARONIEWICZ, Z.: 'Effect of short HV pulses on bacteria and fungi', IEEE Trans. Dielectr. Electr. Insul., 1995, 2, (3), pp. 418-425

5 LUBICKI, P. CROSS, J.D., JAYARAM, S., MAZUREK, B., and STARONIEWICZ, Z.: 'Inactivation of Yersina enterocolitica gramnegative bacteria using high voltage pulse technique'. Conf. Record of IEEE IAS Annual Meeting, 1995, Vol. 2, pp. 1338-1344

6 MATSUMOTO, Y., SATAKE, T., SHIOJI, N., and SAKUMA, A.: 659

7 JOHNSTONE, P.T., and BODGER, P.S.: 'High voltage disinfection of liquids', Trans. Inst. Prof. Eng. N.Z. Electr./Mech. Chem. Eng. Sect., 1997,24 , (1), pp. $30-35$

8 JOHNSTONE, P.T., and BODGER, P.S.: 'Disinfection of deionised water using AC high voltage', IEE Proc, Sci. Meas. Technol, 2000, 147, (3), pp. 141-144

9 NADKARNI, M.A., and BHAT, S.R.: 'Pulse transformers design and fabrication' (McGraw-Hill, New Delhi, 1985) 